\title{
DIABETES MELLITUS E COVID-19: UMA REVISÃO DA LITERATURA
}

\author{
Luísa Thomas Nascimento; ; João C. Tavares Fogaça²; Minéia Weber Blattes ${ }^{3}$
}

\section{RESUMO}

No final de 2019, observou-se um crescente número de pacientes com pneumonia em Wuhan, na China, que rapidamente espalhou-se pelo mundo. Esta doença é causada por um novo tipo de coronavírus, o SARS-CoV-2, e ficou conhecida como Covid-19. A Covid-19 tem causado muitas preocupações devido ao seu alto grau de contágio e complicações associadas. Assim como nas demais doenças infecciosas, o diabetes mellitus (DM) contribui para uma pior evolução da Covid-19. Deste modo, foi realizada uma revisão da literatura com o propósito de encontrar materiais relevantes e atualizados sobre as complicações da Covid-19 em pacientes com DM. Ficou evidente que pacientes com DM que contraem a infecção pelo Sars-Cov-2, apresentam maiores riscos de descompensação do seu quadro metabólico de base, além de aumentar a morbimortalidade pela infecção pelo Sars-Cov-2. Fica claro, assim, o aumento da suscetibilidade dos pacientes com DM a casos graves da infecção pelo SARS-CoV-2.

Palavras-chave: Complicações; Doença metabólica; SARS-CoV-2.

Eixo Temático: Atenção Integral e Promoção à Saúde

\section{INTRODUÇÃO}

No final de 2019, evidenciou-se um surto de pneumonia em Wuhan, na China, que rapidamente se espalhou pelo mundo, tornando-se uma pandemia. Esta doença, causada por um vírus denominado SARS-CoV-2, ficou conhecida como Covid-19. A Covid-19 é uma doença respiratória infecciosa causada por um tipo recém descoberto de coronavírus, responsável por um grande espectro clínico, desde casos assintomáticos até infecções graves e morte.

Assim como nas demais doenças infecciosas, o diabetes mellitus (DM) tem uma grande contribuição na severidade dos casos associados à Covid-19. Em um

\footnotetext{
${ }^{1}$ Acadêmica do Curso de Medicina - Universidade Franciscana/UFN - luisa.thomas@ufn.edu.br

${ }^{2}$ Acadêmico do Curso de Medicina - Universidade Franciscana/UFN - joao.fogaca@ufn.edu.br

${ }^{3}$ Orientadora. Professora do Curso de Biomedicina - Universidade Franciscana/UFN mweber@ufn.edu.br
} 
estudo realizado em Wuhan, em 2020, envolvendo 191 pacientes hospitalizados com Covid-19, $48 \%$ dos pacientes apresentavam comorbidades, sendo a hipertensão a mais comumente encontrada, presente em $38 \%$ da população analisada, seguido de DM em 19\% (ZHOU et al., 2020).

Atualmente, as evidências disponíveis mostram que pacientes com DM, com hiperglicemia fora do alvo, que contraem a Covid-19 estão mais propensos à progressão da doença, podendo levar a resultados fatais. Por este motivo, este artigo de revisão tem como objetivo a análise de dados de estudos publicados sobre o acometimento de Covid-19 e as complicações em pessoas com o diagnóstico de DM.

\section{METODOLOGIA}

Foi realizada uma revisão da literatura com o propósito de encontrar materiais relevantes e atualizados sobre as complicações da Covid-19 em pacientes com DM. A pesquisa bibliográfica foi realizada através de uma revisão da literatura utilizando os bancos de dados: Pubmed, Scielo e Google Acadêmico. Para efetuar a pesquisa, foram utilizados os seguintes descritores "Diabetes mellitus", "COVID-19" e "complicações", e suas variantes em inglês. Como critério de inclusão foram utilizadas publicações dos últimos 3 anos, salvo artigos conceituais relevantes para a temática. Foram excluídos da análise textos que, após leitura do resumo, não abordavam o tema em estudo, apesar de conterem os descritores.

\section{RESULTADOS E DISCUSSÕES}

Em 31 de dezembro de 2019, a China notificou à Organização Mundial da Saúde (OMS) casos de pneumonia em Wuhan, província de Hubei, China, causada por um novo coronavírus, atualmente denominado SARS-CoV-2 (PHELAN, KATZ, GOSTIN, 2020). A doença causada por este vírus ficou conhecida como Covid-19. Em pouco tempo esta doença disseminou-se por diversos países, tornando-se uma pandemia. Os sintomas mais frequentes da Covid-19 são: febre, tosse, dor de 
garganta, dor "tipo sinusite", náuseas, perda de apetite, perda ou alteração do olfato e/ou do paladar, cansaço, dores musculares, dor torácica e falta de ar. Alguns pacientes apresentam sintomas gastrointestinais como náuseas, dor abdominal ou diarreia (Sociedade Brasileira de Infectologia, 2020). Sabe-se que o vírus tem alta transmissibilidade e provoca uma síndrome respiratória aguda que varia de casos leves - cerca de $80 \%$ - a casos muito graves com insuficiência respiratória - entre $5 \%$ e $10 \%$ dos casos. Sua letalidade varia, principalmente, conforme a faixa etária e condições clínicas associadas (Portal UNASUS, 2020). Os principais fatores de risco para evoluir para Covid-19 grave são: pessoas com 60 anos ou mais, doença pulmonar obstrutiva crônica, doença cardiovascular, DM tipo 2 (DM2), obesidade, doença renal crônica, imunocomprometidos e anemia falciforme (Sociedade Brasileira de Infectologia, 2020).

O DM2 é uma doença caracterizada pela resistência insulínica, associada a um mau metabolismo dos carboidratos e consequente hiperglicemia. Como um distúrbio metabólico importante, o DM2 costuma diminuir a resposta imunológica além de apresentar um aumento da resposta inflamatória destes pacientes (LIM et al., 2021)

No DM2, caracterizado por resistência à insulina e forte associação à obesidade, ocorre aumento da secreção de fatores pró-inflamatórios e citocinas por ativação crônica da resposta inata (DONATH et al., 2019) Ambas as condições, DM1 e DM2, podem estar associadas à resposta imune exacerbada identificada em pacientes com DM e COVID-19 (GUPTA et al., 2020). Já está bem documentado que a população com DM apresenta maior risco de admissão hospitalar quando adquire pneumonia, especialmente quando apresenta uma glicemia não controlada. Pacientes com Covid-19 que apresentam hiperglicemia descontrolada têm mostrado internações mais prolongadas e aumento da mortalidade em comparação com pacientes que não apresentam hiperglicemia (TANG et al., 2020). Além disso, não é somente a hiperglicemia que pode ser marcador da gravidade nestes doentes, mas episódios de hipoglicemia e variabilidade glicêmica ampla mostraram-se fatores de 
risco independentes para a mortalidade (LIM et al., 2021). O mau controle glicêmico prejudica vários aspectos do sistema inato e resposta imune adaptativa a infecções virais e à potencial infecção bacteriana secundária nos pulmões (KORNUM et al., 2008)

A glicotoxicidade, o dano endotelial por inflamação, o estresse oxidativo e a produção de citocinas, presentes nos pacientes diabéticos e nos pacientes com Covid-19, contribuem para um risco aumentado de complicações tromboembólicas e de danos a órgãos vitais em pacientes com DM (TANG et al., 2020). Os medicamentos frequentemente usados no tratamento clínico de pacientes com Covid-19, como corticosteróides sistêmicos ou agentes antivirais também podem contribuir para o agravamento da hiperglicemia (LIM et al., 2021). Ademais, os pacientes com DM tendem a apresentar comorbidades associadas, como obesidade, hipertensão, doença arterial coronariana e doença renal crônica, as quais podem piorar ainda mais o prognóstico de Covid-19.

A doença grave, manifestada como necessidade de hospitalização, intubação e morte, pode ocorrer em indivíduos saudáveis de qualquer idade, mas o risco de doença grave é mais pronunciado em adultos com idade avançada ou comorbidades médicas subjacentes, como o DM (YANG et al., 2020).

Comparado com o peso normal, obesidade foi significativamente e independentemente associada com riscos aumentados de pneumonia grave e exigindo oxigenoterapia hospitalar (CAO et al., 2021). O papel do excesso de peso e obesidade em Covid-19 pode estar relacionada a dificuldade de ventilação e oxigenação, pois a obesidade é um fator de risco conhecido para ventilação anormal e pode contribuir para a redução da capacidade funcional residual pulmonar e complacência da da parede torácica (CHIUMELLO et al., 2016).

Segundo Fadini e colaboradores, as evidências disponíveis mostram que a presença de DM em pacientes com Covid-19, torna-os mais propensos à progressão da doença e resultados fatais. Assim, observa-se que o DM pode não aumentar o 
risco de infecção por SARS-CoV-2, mas pode piorar o resultado da doença no paciente (FADINI et al., 2020).

Em um estudo publicado no New England Journal Medicine por Guan et al. em 2020, que analisou 1099 pacientes com diagnóstico de Covid-19, 81 (7,4\%) apresentaram diagnóstico de DM. Do total de pacientes analisados, 173 apresentavam doença severa e 5,7\% destes pacientes apresentavam DM (GUAN, et al., 2020).

Outro estudo analisou a mortalidade de 52 pacientes criticamente doentes com Covid-19 em Wuhan. Do total, 32 pacientes vieram a óbito e $22 \%$ destes apresentavam DM (YANG et al., 2020). Assim, verifica-se que a porcentagem de história conhecida de DM2 foi significativamente maior entre os pacientes que vieram a óbito por infecção respiratória do que aqueles que sobreviveram (BOOTH et al., 2003).

Os pacientes com Covid-19 com doenças subjacentes, como DM, possuem chance seis vezes maiores de serem hospitalizados e doze vezes mais chances de morrer (STOKES et al., 2020). Além disso, a própria infecção pelo SARS-CoV-2 aumenta as complicações pré-disponentes em pacientes com DM, como desregulação da glicose e predisposição a complicações como outras infecções, coma hiperosmolar, cetoacidose e até mesmo eventos cardíacos agudos (GUPTA et al., 2020)

Segundo Gonzalez et al. (2011), pacientes com DM são mais suscetíveis a desenvolver estresse psicológico, ansiedade e depressão. O estresse em pacientes com DM está associado a pior controle metabólico, que inclui maior nível de hemoglobina glicada, maior índice de massa corporal e pressão arterial elevada. (GONZALES et al., 2011). Fatores estressantes são susceptíveis ao aumento das taxas de ansiedade e depressão, o que também pode levar a uma má adesão de medicamentos e agravamento dos fatores de riscos (GRENARD et al., 2011). Um estudo desenvolvido por Franco e colaboradores mostrou que a disponibilidade reduzida de equipes multidisciplinares e a maior dificuldade em obter cuidados 
médicos durante a pandemia podem interferir diretamente na adesão ao tratamento (FRANCO et al., 2021). Ademais, dois outros estudos mostram o aumento nos transtornos psicológicos e alimentares durante a quarentena, o que pode levar a um impacto ainda maior a longo prazo em pacientes com DM, impactando negativamente na adesão do comportamento de autocuidado destes pacientes (BROOKS et al., 2021; VINDEGAARD e BENROS, 2020).

\section{CONCLUSÃO}

Observa-se assim, portanto, a correlação entre duas grandes pandemias mundiais, o DM e a Covid-19. Fica claro o aumento da suscetibilidade dos pacientes com diagnóstico de DM a casos graves da infecção pelo SARS-CoV-2. Além de apresentarem maiores comorbidades associadas, os pacientes diabéticos apresentam um perfil inflamatório crônico, predispondo a complicações. O estado hiperglicêmico e a liberação de citocinas inflamatórias nestes pacientes são os principais fatores associados aos casos graves, como internações, uso de ventilação invasiva e até mesmo óbito nos pacientes com DM.

Ademais, a própria infecção e os aspectos sociais trazidos pela pandemia do Covid-19 afetam diretamente estes pacientes. Pacientes com DM que contraem a infecção pelo SARS-CoV-2, apresentam maiores riscos de descompensação metabólica e o isolamento social consequente da nova pandemia apresenta risco elevado de quadros de ansiedade e depressão, os quais auxiliam no mau controle da doença metabólica e aumento dos riscos.

\section{AGRADECIMENTOS}

Agradeço ao Grupo Multidisciplinar de Educação em Diabetes da Universidade Franciscana (GEPED) pela oportunidade.

\section{REFERÊNCIAS}

BOOTH, C.M. et al. Clinical features and short-term outcomes of 144 patients with SARS in the greater Toronto area. JAMA, v. 289, n. 21, p. 2801-9, 2003. 
BROOKS, S. K. et al. The psychological impact of quarantine and how to reduce it: rapid review of the evidence. Lancet, v. 395, p. 912-20, 2020.

CAO, P. et al. Obesity and COVID-19 in Adult Patients With Diabetes. Diabetes, v. 70, n. 5, p. 1061-1069, 2021.

CHIUMELLO, D et al. Effect of body mass index in acute respiratory distress syndrome. British journal of anaesthesia, v. 116, n. 1, p. 113-21, 2016.

DONATH, $\mathrm{M}$ et al. Targeting innate immune mediators in type 1 and type 2 diabetes. Nature reviews. Immunology, v. 19, n.12, p. 734-746, 2020.

FADINI, G.P. et al. Prevalence and impact of diabetes among people infected with SARS-CoV-2. J Endocrinol Invest, v.43, p. 867-869, 2020.

FRANCO, D.W. et al. Medical adherence in the time of social distancing: a brief report on the impact of the COVID-19 pandemic on adherence to treatment in patients with diabetes. Arch Endocrinol Metab, 2021.

GONZALEZ, J. et al. Depression in diabetes: have we been missing something important?. Diabetes care, v. 34, n. 1, p. 236-9, 2011.

GRENARD, J.L. et al. Depression and medication adherence in the treatment of chronic diseases in the United States: a meta-analysis. Journal of general internal medicine, v. 26, p.1175-82, 2011.

GUAN W., NI Z., HU Y. et al. Clinical Characteristics of Coronavirus Disease 2019 in China. J Emerg Med, v. 58, n.4, p.711-712, 2020. 
GUPTA, R., HUSSAIN, A., MISRA, A. Diabetes and COVID-19: evidence, current status and unanswered research questions. Eur J Clin Nutr, v.74, p. 864-870, 2020.

GUPTA R. et al. Clinical considerations for patients with diabetes in times of COVID-19 epidemic. Diabetes Metab Syndr, v.14, n.3, p.211-212, 2020.

KORNUM, J. et al. Diabetes, glycemic control, and risk of hospitalization with pneumonia: a population-based case-control study. Diabetes care, v. 31,8, p.1541-5, 2008.

LIM, S. et al. COVID-19 and diabetes mellitus: from pathophysiology to clinical management. Nature reviews. Endocrinology, v. 17,1, p. 11-30, 2021.

PHELAN A.L., KATZ R., GOSTIN, L.O. The Novel Coronavirus Originating in Wuhan, China: Challenges for Global Health Governance. JAMA, v. 323, n.8, p. 709-710, 2020.

SOCIEDADE BRASILEIRA DE INFECTOLOGIA. Atualizações e recomendações sobre a Covid-19 (2020). $<$ https://infectologia.org.br/wp-content/uploads/2020/12/atualizacoes-e-recomendacoe

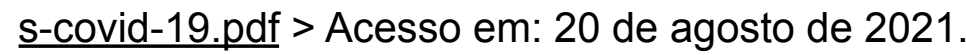

STOKES, E.K. et al. Coronavirus Disease 2019 Case Surveillance — United States, January 22-May 30, 2020. MMWR and Morbidity and Mortality Weekly Report, v. 69, p. 759-765, 2020. 
TANG, N. et al. Abnormal coagulation parameters are associated with poor prognosis in patients with novel coronavirus pneumonia. Journal of thrombosis and haemostasis, v. 18,4, p. 844-847, 2020.

UNASUS. Protocolo de Manejo Clínico do Coronavírus (Covid-19) na atenção primária à saúde. Ministério da Saúde (Secretaria de Atenção Primária à Saúde), Brasília, 2020.

Disponível em

https://saude.rs.gov.br/upload/arquivos/202004/14140606-4-ms-protocolomanejo-aps -ver07abril.pdf> Acesso em: 22 de agosto de 2021.

VINDEGAARD, N.; BENROS, M. E. COVID-19 pandemic and mental health consequences: Systematic review of the current evidence. Brain Behav Immun., v. 89, p. 531-42, 2020.

YANG, X. et al. Clinical course and outcomes of critically ill patients with SARS-CoV-2 pneumonia in Wuhan, China: a single-centered, retrospective, observational study. The Lancet Respiratory Medicine, v. 8,5, p. 475-481, 2020.

ZHOU, F. et al. Clinical course and risk factors for mortality of adult inpatients with COVID-19 in Wuhan, China: a retrospective cohort study. Lancet, v. 395, p.1054-1062, 2020. 